M. Beltrametti and M. Palleschi

Nagoya Math. J.

Vol. 101 (1986), 27-36

\title{
ON THREEFOLDS WITH LOW SECTIONAL GENUS
}

\author{
MAURO BELTRAMETTI AND MARINO PALLESCHI
}

\section{Introduction}

The general problem of rebuilding the threefolds $X$ endowed with a given ample divisor $H$, possibly non-effective, is closely related to the study of the complete linear system $\left|K_{X}+H\right|$ adjoint to $H$. Many powerful results are known about $\left|K_{X}+H\right|$, for instance when the linear system $|H|$ contains a smooth surface or, more particularly, when $H$ is very ample (e.g. see Sommese [S1] and [S2]). From this point of view we study some properties of $\left|K_{X}+H\right|$, which turn out to be very useful in the description of the threefolds $X$ polarized by an ample divisor $H$ whose arithmetic virtual genus $g(H)$ is sufficiently low.

In section 1 we recall some preliminaries.

In section 2 an explicit description of the threefolds on which the sheaf $\mathcal{O}_{X}\left(n\left(K_{X}+H\right)\right)$ fails to be spanned by its global sections for $n \gg 0$ is pointed out (Theorem 2.2). This is a consequence of a part of Mori's theory on extremal rays. In brief this theorem assures that it is always possible to assume $\mathcal{O}_{X}\left(n\left(K_{X}+H\right)\right)$ to be spanned for $n \gg 0$ up to contractions of (-1)-planes, apart from some classes of threefolds, which are fully described. Incidentally this answers a question put by Sommese in [S2].

In section 3 first of all we characterize the pairs $X, H$ in the cases $g(H)=0$ and $g(H)=1$ (Propositions 3.1 and 3.2), in this way recovering some classical results (cf. [E]; see also [Ro], IV, §9) in the wider context of ample divisors. As far as higher values of $g(H)$ are concerned, the pairs $X, H$ are classified under the assumption $H^{3} \geq 2 g(H)-2$ (Theorem 3.3). This analysis together with the general theory of Fano threefolds leads to the explicit description of $X, H$ where $g(H)=2, H^{3} \geq 2$ (Proposition 3.5). This is again linked to a classical piece of research carried out by Enriques in [E].

Received July 27, 1983.

Revised February 27, 1985. 
The authors wrote this paper while they were Visiting Professors at Warwick University, and they would like to thank the Mathematics Department for their hospitality.

They are also grateful to the referee for suggesting valuable improvements.

\section{$\S 1$. Notation and preliminaries}

Consider a $n$-dimensional nonsingular projective variety $\left(V, \mathcal{O}_{V}\right)$ over an algebraic closed field $k$ of characteristic zero. When $n=3$ or 2 , such a $V$ will be called threefold or surface respectively.

Any canonical divisor of $V$ will be denoted by $K_{V}$. The dimension $q(V)$ of the $k$-vector space $H^{1}\left(V, \mathcal{O}_{V}\right)$ will be said irregularity of $V$. If $D$ is a divisor on $V$, the complete linear system determined by $D$ will be denoted by $|D|$ and we shall write $D_{\mid Y}$ for the restriction divisor of $D$ to a subvariety $Y$ of $X$. We shall also put

$$
N_{q}(V)=(\{q \text {-cycles }\} / \approx) \otimes R,
$$

where "§" means numerical equivalence. We shall express the intersection of cycles by the symbol ".", and the linear equivalence of divisors by "三". The dimension $\rho(V)$ of the real vector space $N_{1}(V)$ will be called Picard number of $V$. Morever $\overline{N E}(V)$ will be the closure of the convex cone $N E(V) \subset N_{1}(V)$ generated by effective 1-cycles, with respect to any Euclidean norm.

Let $(X, H)$ be a nonsingular polarized threefold. We say that an irreducible divisor $D$ on $X$ is a $(-1)$-plane if $D \cong P^{2}$, and $\mathcal{O}_{D}(-D) \cong \mathcal{O}_{D}(H)$ $\cong \mathcal{O}_{P^{2}}(1)$. The pair $(X, H)$ (or $X$ if there is no danger of confusion) is said to be a minimal model when it does not contain any $(-1)$-planes.

We say that a polarized threefold $(Y, M)$ is a $(-1)$-contraction of $(X, H)$ if there exists a morphism $\Phi: X \rightarrow Y$ which decomposes as $X=$

$X_{1} \stackrel{\phi_{1}}{\longrightarrow} X_{2} \rightarrow \cdots \stackrel{\phi_{s}}{\longrightarrow} X_{s+1}=Y(s \geqq 0)$ such that $\phi_{i}$ is the blowing up of some point $p_{i+1} \in X_{i+1}, D_{i}=\phi_{i}^{-1}\left(p_{i+1}\right)$ is a $(-1)$-plane of $\left(X_{i},\left(\phi_{i-1} \circ \cdots \circ \phi_{1}\right)_{*} H\right)$, $(Y, M)$ is a minimal model and, if $s \geqq 1$,

$$
H=\Phi^{*} M-\sum_{i=1}^{s}\left(\phi_{i-1} \circ \cdots \circ \phi_{1}\right)^{*} D_{i},
$$

where of course in the summation index $i=1$ is meant to give $D_{1}$.

We note that if $f: X \rightarrow Y$ is a blowing up at one point $p$ then $M=f_{*} H$ 
is an ample divisor because the base locus of some multiple of $M$ is contained in $\{p\}$ and $M$ is numerically positive. It is clear that $(X, H)$ has a $(-1)$-contraction and uniqueness follows from the following

Proposition 1.1. Let $(X, H)$ be a polarized threefold and let $f: X \rightarrow Z$ be a contraction of $a(-1)$-plane $E$ of $(X, H)$. Then an arbitrary $(-1)$ contraction $(Y, M)$ of $(X, H)$ is also a (-1)-contraction of $\left(Z, f_{*} H\right)$. In particular, a (-1)-contraction is unique up to isomorphism.

Proof. We use induction on $\rho(X)-\rho(Y)$. In the proof, we use the notation introduced in defining $(-1)$-contractions. Then $s=\rho(X)-\rho(Y)$. If $s=0$, then the assertion holds since $(X, H)$ has no $(-1)$-planes. Let $s \geqq 1$. If $D_{1} \cap E \neq \emptyset$, then every curve in $D_{1} \cup E$ is contracted by both $f$ and $\phi_{1}$ because $D_{1} \cong E \cong \boldsymbol{P}^{2}$. Thus $f=\phi_{1}$ and the assertion is clear in this case. Assume now that $D_{1} \cap E=\emptyset$. Then $f\left(D_{1}\right)$ (resp. $\phi_{1}(E)$ ) is a (-1)-plane of $Z$ (resp. $X_{2}$ ), and the contraction $V$ of $(-1)$-plane $f\left(D_{1}\right)$ of $Z$ is also the contraction of $(-1)$-plane $\phi_{1}(E)$ of $X_{2}$. Since $(Y, M)$ is a $(-1)$-contraction of $\left(X_{2}, \phi_{1 *} H\right)$ and $\rho\left(X_{2}\right)-\rho(Y)=s-1$, the assertion follows by induction.

q.e.d.

We shall say that $X$ is a $\left(Q \subset P^{3}\right)$-bundle if $X$ is embedded in a $\boldsymbol{P}^{3}$-bundle $\mathscr{P}$ over a nonsingular curve $Y$ such that every fibre $X_{y}$ is an irreducible reduced quadric of $\boldsymbol{P}^{3} \simeq \mathscr{P}_{y}, y \in Y$.

Fano threefolds will often occur throughout the paper. We shall always denote by $\mathscr{L}$ the unique (up to linear equivalence) ample divisor on $X$ such that $-K_{X} \equiv r \mathscr{L}$, where $r$ is the index of $X$. The integer $d=H^{3}$ will be called the degree of $X$ (see also [Mu], $\S 1$ ).

A part of Mori's theory of extremal rays is to be used throughout the paper. As far as definition and generalities about numerically effective cycles (nef cycles from now on), extremal rays, extremal rational curves, etc. are concerned, we shall refer to Mori's papers [M1], [M2].

\section{§2. On the system adjoint to an ample divisor}

To begin with, let us state the following

LEMMA 2.1. Let us consider an ample divisor $H$ on a threefold $X$. If $K_{X}+H$ is not nef then there exist an extremal ray $R$, a projective variety $Y$ and a morphism $\Phi=\operatorname{cont}_{R}: X \rightarrow Y$ unique up to isomorphism, such that $\left(K_{X}+H\right) \cdot C<0$ for any irreducible curve $C \subset X$ which is contracted by $\Phi$. 
Proof. Since $K_{X}+H$ is not nef, one has

$$
\left(K_{X}+H\right) \cdot \gamma<0
$$

for a suitable curve $\gamma \subset X$. After choosing $\varepsilon \in R, 0<\varepsilon<1$, in view of Mori's theorem on cone ([M1], 1.4.2) it is possible to write $\gamma \approx \sum \lambda_{i} \ell_{i}+\Gamma$ where $\lambda_{i} \in R, \lambda_{i} \geq 0$, each $\ell_{i}$ is an extremal rational curve, $\Gamma \in \overline{N E}(X)$ and $K_{X} \cdot \Gamma \geq-\varepsilon(\Gamma \cdot H)$. Since $H \cdot \Gamma \geq 0$ by Kleiman ampleness criterion [K], it is possible to find an extremal rational curve $\ell_{j}$ such that

$$
\left(K_{X}+H\right) \cdot \ell_{j}<0 .
$$

Therefore $\left(K_{X}+H\right) \cdot C<0$ for every irreducible curve $C$ whose class [C] in $N_{1}(X)$ belongs to the extremal ray $R=R_{+}\left[\ell_{j}\right]$. On the other hand there exist a projective variety $Y$ and a morphism $\Phi: X \rightarrow Y$ such that an irreducible curve $C$ is contracted by $\Phi$ if and only if [C] $\in R$ ([M1] 3.1).

q.e.d.

The following theorem play an important role in the forthcoming analysis and has also interest in itself (compare with Question B put by Sommese in [S2]). Actually it might well be considered as a known property, since it is a direct consequence of Mori's theory of extremal rays. Nevertheless we sketch its proof for readers' convenience, because we do not know any reference for it. As to the following proof we will use two recent results from Kawamata [Ka] and Reid [R] so as to avoid a rather long analysis of the extremal rays occurring when $K_{X}+H$ is nef.

Theorem 2.2. Let us consider an ample divisor $H$ on a threefold $X$. If $\mathcal{O}_{X}\left(n\left(K_{X}+H\right)\right)$ is not spanned by its global sections for $n \gg 0$, then either (2.2.1) $X$ is a $P^{1}$-bundle and $\mathcal{O}_{F}(H) \simeq \mathcal{O}_{P^{1}}(1)$ for every fibre $F$;

(2.2.2) $X$ is a $P^{2}$-bundle and $\mathcal{O}_{F}(H) \simeq \mathcal{O}_{P^{2}}(n), n=1,2$, for every fibre $F$;

(2.2.3) $X$ is $a\left(Q \subset P^{3}\right)$-bundle with $\rho(X)=2$ and $\mathcal{O}_{Q}(H)=\mathcal{O}_{Q}(1)$ for every fibre $Q$;

(2.2.4) $X$ is a Fano threefold with $\rho(X)=1$, of index $r \geqslant 2$ and $H \equiv n \mathscr{L}$, $n<r$; or

(2.2.5) there exist a unique, up to isomorphism, (-1)-contraction $Y, M$ of $X, H$ and either $Y, M$ is as in one of the previous cases or $\mathcal{O}_{Y}\left(n\left(K_{Y}+M\right)\right)$ is spanned by its global sections for $n \gg 0$.

Proof. First let us suppose $K_{X}+H$ to be nef. Then the Kodaira dimension $\kappa\left(X, K_{X}+H\right)$ is not negative as has recently been proved by 
M. Reid [R]. Therefore Theorem 6 in [Ka] says that $\mathcal{O}_{X}\left(n\left(K_{X}+H\right)\right)$ is spanned by its global sections for $n \gg 0$. So we can assume that $K_{X}+H$ is not nef and consider both the extremal ray $R$ and the morphism $\Phi: X \rightarrow Y$ as in Lemma 2.1 .

Let us start analysing the case when $R$ is nef. Then $Y$ is nonsingular and one of the cases listed in [M1], 3.5 holds. Let $F$ be the general fibre of $\Phi$. The conic bundle case leads to (2.2.1). Indeed, by Lemma 2.1, we get

$$
0>\left(K_{X}+H\right) \cdot F=\operatorname{deg} K_{F}+H \cdot F=-2+H \cdot F,
$$

so $H \cdot F=1$, which is enough to conclude. In the Del Pezzo fibering case Lemma 2.1 gives

$$
0>\left(K_{X}+H\right) \cdot \gamma=\left(K_{F}+H_{\mid F}\right) \cdot \gamma
$$

for every irreducible curve $r \subset F$. Now $F$ is a Del Pezzo surface, then Theorem 1.2 in [M2] assures that the class [ $\gamma$ ] of $\gamma$ in $N E(F)$ is generated by extremal rational curves of $F$. Therefore $(*)$ implies the existence of an extremal rational curve $C \subset F$ such that $\left(K_{F}+H_{\mid F}\right) \cdot C<0$. Then $K_{F} \cdot C \leq-2$ and so $C$ is not an exceptional curve of the first kind. Therefore either $F \simeq P^{2}$ or $F$ is a $P^{1}$-bundle (cf. [M1], 2.1). In the former case $X$ is a $\boldsymbol{P}^{2}$-bundle (cf. [M2], 2.6) and by taking an effective generator of $\operatorname{Pic}(F)$ as $\gamma$, relation $\left(^{*}\right)$ implies $\mathcal{O}_{F}(H) \simeq \mathcal{O}_{P^{2}}(n), n=1,2$, so we have (2.2.2). In the latter case $F$ is isomorphic to $\boldsymbol{P}^{1} \times \boldsymbol{P}^{1}$. Then $X$ is a $\left(Q \subset P^{3}\right)$-bundle ([M2], 2.6) and again $\left(^{*}\right)$ gives (2.2.3). Finally we have to consider the case when $X$ is a Fano threefold of index $r$ with $\rho(X)=1$. Then $K_{X} \equiv-r \mathscr{L}$ and $H \equiv n \mathscr{L}$ with $n<r$, as $K_{X}+H$ is not nef, which gives (2.2.4).

We must now consider the case when $R$ is not nef. In this case $\Phi$ is a birational morphism contracting a unique irreducible divisor $D$ (see [M1], 3.3). Moreover one sees that $K_{X} \cdot R \leq-2$ in view of Lemma 2.1. Therefore a standard computation shows that the three 'singular contraction" cases and the "contraction of a scroll" case in [M1] 3.3 do not occur, so the only possible case is when $Y$ is nonsingular, $\Phi(D)$ is a point, $D \simeq \boldsymbol{P}^{2}$ and $\mathcal{O}_{D}(D) \simeq \mathcal{O}_{P^{2}}(-1)$. Let us consider the ample divisor $M=\Phi_{*} H$. By repeating the same reasoning as above on $Y$, we can assume $K_{Y}+M$ to be nef. Then $\mathcal{O}_{Y}\left(n\left(K_{Y}+M\right)\right)$ is spanned by its global sections for $n \gg 0$ in view of [Ka] and [R].

q.e.d. 
Let us point out the following easy consequence of Theorem 2.2, which will be useful in the sequel.

Lemma 2.3. Let $A$ be an ample divisor on a threefold $X$. If $K_{X}+2 A$ is not nef, then either

(2.3.1) $X$ is a $P^{2}$-bundle and $\mathcal{O}_{F}(A) \simeq \mathcal{O}_{P^{2}}(1)$ for every fibre $F$;

(2.3.2) $X \simeq P^{3}$ and $\mathcal{O}_{X}(A) \simeq \mathcal{O}_{P^{3}}(1)$, or

(2.3.3) $X \simeq Q \subset P^{4}, Q$ quadric hypersurface and $\mathcal{O}_{X}(A) \simeq \mathcal{O}_{Q}(1)$.

Proof. Let us put $H=2 A$ and suppose that $K_{X}+H$ is not nef. Then the pair $X, H$ belongs to one of the classes listed in Theorem 2.2. On the other hand $H \cdot \gamma \geq 2$ for every curve $\gamma$ in $X$ and so (2.2.1), (2.2.2) with $n=1$, and (2.2.3) are excluded. If (2.2.4) holds, then $A \equiv m \mathscr{L}$ with $2 m<r$ so that $X$ is a Fano threefold of index $r \geq 3$, which gives (2.3.2) and (2.3.3) (see also [Mu], 2.1). Finally, if (2.2.5) holds, pick up a (-1)plane $D \subset X$ and consider the effective generator $B$ of Pic $(D)$. We know that $\mathcal{O}_{D}(D) \simeq \mathcal{O}_{P^{2}}(-1)$ and so

$$
\left(K_{X}+H\right) \cdot B=\left(K_{D}-D+H_{\mid D}\right) \cdot B=-2+2 A \cdot B \geq 0,
$$

which contradicts Lemma 2.1 and completes the proof.

q.e.d.

\section{$\S 3$. Threefolds with a given sectional genus}

We are going to study the threefolds $X$ endowed with an ample divisor $H$ with a given (arithmetic virtual) genus $g(H)$, defined by the relation

$$
2 g(H)-2=\left(K_{X}+2 H\right) \cdot H^{2} .
$$

In case when $|H|$ contains smooth members $H_{1}$ and $H_{2}$ such that $C=H_{1} \cdot H_{2}$ is a nonsingular curve, then $g(H)$ is merely the arithmetic genus of the curve $C$. In the general case, $g(H)$ is a non-negative integer for the following reasons:

(i) $g(H) \in Z$ : in fact, for sufficiently large odd $n, n H$ is very ample and $g(n H) \in N$ as seen above. Since $\left(K_{X}+2 n H\right) \cdot n^{2} H^{2} \equiv\left(K_{X}+2 H\right) \cdot H^{2}$ $\bmod 2$, one sees that $g(H) \in Z$;

(ii) $g(H) \geqq 0$ : since $g(H) \geqq 0$ in case $K_{X}+2 H$ is nef or $H$ is very ample, one has only to check $g(H) \geqq 0$ in case (2.3.1) by Lemma 2.3. Let $\pi: X \rightarrow C$ be the $P^{2}$-bundle morphism. Then $H+\pi^{*} M$ is very ample for some ample line bundle $M$ of $C$ and a direct calculation shows that $g(H)=g\left(H+\pi^{*} M\right)$, which is non-negative. 
Proposition 3.1. If $g(H)=0$, then either

(3.1.1) $X$ is a $P^{2}$-bundle and $\mathcal{O}_{F}(H) \simeq \mathcal{O}_{P^{2}}(1)$ for every fibre $F$;

(3.1.2) $X \simeq P^{3}$ and $\mathcal{O}_{X}(H) \simeq \mathcal{O}_{P 3}(1)$; or

(3.1.3) $X \simeq Q \subset P^{4}, Q$ quadric hypersurface, and $\mathcal{O}_{X}(H) \simeq \mathcal{O}_{Q}(1)$.

Proof. Relation (3.0.1) reads $\left(K_{X}+2 H\right) \cdot H^{2}=-2$, then $K_{X}+2 H$ is not nef. Thus the assertion follows from Lemma 2.3 . q.e.d.

Proposition 3.2. If $g(H)=1$, then either

(3.2.1) $X$ is a $P^{2}$-bundle and $\mathcal{O}_{F}(H) \simeq \mathcal{O}_{P^{2}}(1)$ for every fibre $F$;

(3.2.2) $X \simeq P^{3}$ and $\mathcal{O}_{X}(H) \simeq \mathcal{O}_{P^{3}}(2)$; or

(3.2.3) $X$ is a Fano threefold of index $r=2$, degree $d, K_{X} \equiv 2 H$, and $1 \leq d=H^{3} \leq 7$.

Proof. Relation (3.0.1) reads $\left(K_{X}+2 H\right) \cdot H^{2}=0$. Let us assume $K_{X}+$ $2 H$ to be nef. Then $\left(K_{X}+2 H\right)^{2} \in \overline{N E}(X)$ and so $H \cdot\left(K_{X}+2 H\right)^{2} \geq 0$ ([K], III). Since $H^{3}>0$ one sees that $K_{X}+2 H$ is numerically trivial by [K], I, Section 4, Prop. 3. Therefore $-K_{X}$ is ample, so $q(X)=0$ and Pic $(X)$ is torsion free (cf. [Mu], 1.11). It follows $K_{X} \equiv 2 H$ and $X$ is a Fano threefold of index $r=2,4$. If $r=2$ it is known that $1 \leq H^{3} \leq 7$ (cf. [Mu], 2.2) so we get (3.2.3), while $r=4$ gives (3.2.2).

Whenever $K_{X}+2 H$ is not nef we obtain (3.2.1) by Lemma 2.3. q.e.d.

As far as higher values of $g(H)$ are concerned, we can assume $g(H) \geq 2$ after Propositions 3.1 and 3.2. We can prove

TheOREm 3.3. Let $H$ be an ample divisor on the threefold $X$ and let us assume $H^{3} \geq 2 g(H)-2, g(H) \geq 2$. Then either

(3.3.1) $X$ is a $\boldsymbol{P}^{1}$-bundle, $\mathcal{O}_{F}(H) \simeq \mathcal{O}_{P^{1}}(1)$ for each fibre $F$;

(3.3.2) $X$ is a $P^{2}$-bundle, $\mathcal{O}_{F}(H) \simeq \mathcal{O}_{P^{2}}(n), n=1,2$ for each fibre $F$;

(3.3.3) $X$ is a $\left(Q \subset P^{3}\right)$-bundle, $\mathcal{O}_{Q}(H) \simeq \mathcal{O}_{Q}(1)$ for each fibre $Q$;

(3.3.4) $H^{3}>2 g(H)-2, X \simeq \boldsymbol{P}^{3}, \mathcal{O}_{X}(H) \simeq \mathcal{O}_{P^{3}}(3)$ and $g(H)=10$;

(3.3.5) $H^{3}>2 g(H)-2, X \simeq Q \subset P^{4}, Q$ quadric hypersurface, $\mathcal{O}_{X}(H) \simeq$ $\mathcal{O}_{Q}(2), g(H)=5$;

$H^{3}=2 g(H)-2, X \simeq \boldsymbol{P}^{3}, \mathcal{O}_{X}(H) \simeq \mathcal{O}_{P^{3}}(4), g(H)=33 ;$

(3.3.7) $H^{3}=2 g(H)-2, X \simeq Q \subset P^{4}, Q$ quadric hypersurface, $\mathcal{O}_{X}(H) \simeq$ $\mathcal{O}_{Q}(3), g(H)=28$;

(3.3.8) $H^{3}=2 g(H)-2, X$ is a Fano threefold of index $r=1,2$ degree $d$, $K_{X} \equiv-H$ and one has furthermore $g(H)=4 d+1, d=1,2, \cdots, 7$ if $r=2$; or 
(3.3.9) there exists a unique (-1)-contraction $Y, M$ of $X, H, g(M)=g(H)$ and the pair $Y, M$ is as in (3.3.1), (3.3.2), (3.3.3), (3.3.4) or (3.3.5) with $M^{3}>2 g(M)-2$.

Proof. Assume $X$ to be a minimal model. First, let us consider the case $H^{3}>2 g(H)-2$. Formula (3.0.1) implies $K_{X}+H$ is not nef and so $X$ belongs to one of the classes listed in Theorem 2.2. Classes (2.2.1), (2.2.2), (2.2.3) give (3.3.1), (3.3.2), (3.3.3) respectively. Now suppose $X$ to be a Fano threefold of index $r \geq 2$ as in (2.2.4). Whenever $r=4,3$ we get (3.3.4), (3.3.5) respectively; while $r=2$ is to be excluded, otherwise we would have $K_{X} \equiv-2 H$ and then $g(H)=1$ in view of (3.0.1). Now assume $H^{3}=2 g(H)-2$. Then formula (3.0.1) gives

$$
\left(K_{X}+H\right) \cdot H^{2}=0 .
$$

Let us start by supposing $K_{X}+H$ nef. Therefore $\left(K_{X}+H\right)^{2} \in \overline{N E}(X)$ and reasoning as in the proof of 3.2 one sees that $K_{X}+H$ is numerically trivial (see again [K]). Thus $X$ is a Fano threefold of index $r$, degree $d$. Hence $q(X)=0$ and Pic $(X)$ is torsion free, so that $K_{X} \equiv-H$. If $r=4,3$ then $X \simeq P^{3}$ or $X \simeq Q \subset P^{4}, Q$ quadric hypersurface, so we get (3.3.6), (3.3.7) respectively. As soon as $r=2$ then $1 \leq d \leq 7$ by [Mu] 2.2; one has $K_{X} \equiv-2 \mathscr{L}$ that is $H \equiv 2 \mathscr{L}$ and (3.3.8) follows. Again, whenever $K_{X}+H$ is not nef, $X$ belongs to one of the classes listed in Theorem 2.2: (2.2.1), (2.2.2), (2.2.3) give (3.3.1), (3.3.2), (3.3.3), while (2.2.4) does not occur. Otherwise we could write $K_{X} \equiv-r \mathscr{L}, H \equiv n \mathscr{L}, n<r$, which would imply

$$
\left(K_{X}+H\right) \cdot H^{2}=(n-r) \mathscr{L} \cdot H^{2}<0
$$

contradicting $(* *)$.

It remains to consider the case when $X$ is not a minimal model. Let $f: X \rightarrow Z$ be the contraction of $a(-1)$-plane $D$ of $(X, H)$, and $N=f_{*} H$. Since $K_{X}+2 H=f^{*}\left(K_{Z}+2 N\right)$, the projection formula gives

$$
\begin{aligned}
2 g(H)-2 & =\left(K_{X}+2 H\right) \cdot H^{2} \\
& =\left(K_{Z}+2 N\right) \cdot f_{*} H^{2}=\left(K_{Z}+2 N\right) \cdot N^{2}=2 g(N)-2,
\end{aligned}
$$

hence $g(N)=g(H)$ and also

$$
H^{3}=\left(f^{*} N-D\right)^{3}=\left(f^{*} N\right)^{3}-D^{3}=N^{3}-\left((D)_{D}\right)^{2}=N^{3}-1 .
$$

Therefore the $(-1)$-contraction $(Y, M)$ of $(X, H)$ satisfies $g(M)=g(H)$ and $M^{3}>2 g(M)-2$, which implies that $K_{Y}+M$ is non nef by definition 
of $g(M)$. Thus Theorem 2.2 applied to $(Y, M)$ gives (3.3.9). $\quad$ q.e.d.

Remark 3.4. Whenever the cycle $H^{2}$ is a nonsingular curve $C$, in $\boldsymbol{P}^{2}$-bundle cases (3.1.1), and (3.3.2) with $n=1$, the curve $C$ is isomorphic to the base curve $Y$. While in the $\left(Q \subset P^{3}\right)$-bundle case (3.3.3) (resp. in (3.3.2) with $n=2$ ) the curve $C$ is a double (resp. quadruple) covering of $Y$.

The previous results lead to a complete description of pairs $X, H$ with genus $g(H)=2$ and $H^{3} \geq 2$. In this particular case we have

Proposition 3.5. Let us suppose $g(H)=2, H^{3} \geq 2$. Then either (3.5.1) $X$ is as in (3.3.1), (3.3.2) or (3.3.3) of Theorem 3.3;

(3.5.2) there exists a unique (-1)-contraction $Y, M$ of $X, H, g(M)=2$ and the pair $Y, M$ is as in (3.5.1); or

(3.5.3) $\quad H^{3}=2$ and $|H|$ gives a double covering $X \rightarrow \boldsymbol{P}^{3}$ with ramification locus a smooth sextic surface.

Proof. Indeed, Theorem 3.3 implies either (3.5.1) and (3.5.2), or that $X$ is a Fano threefold of index $r=1$, degree $d=2$. In this case by looking over the classification of Fano threefolds given in [I1], Section 7, [M-M] we get (3.5.3).

q.e.d.

Remark 3.6. We have no satisfactory results when $g(H)=2, H^{3}=1$. By using the same arguments as above we can only prove that if $K_{X}$ is not numerically trivial, then either (3.5.1) or (3.5.2) holds or the complete linear system $\left|n\left(H+K_{X}\right)\right|, n \gg 0$, gives a morphism $\phi: X \rightarrow Y$, over a curve $Y$ such that the general fibre is a Del Pezzo surface.

The general theory of Fano threefolds, for which we refer to $[\mathrm{Mu}$, leads to the following:

Supplement 3.7. a) The Fano threefolds occurring in (3.2.3), (3.3.8) are classified in [I1], [I2], [M-M]. Moreover as a consequence of one of Shokurov's theorems the linear system $|H|$ contains a Del Pezzo surface or a $K 3$ surface according to whether $r>1$ or $r=1$.

b) In (3.3.8), since $-K_{X} \equiv H$, the integer $g(H)$ is nothing but the genus $g=g\left(-K_{X}\right)$ of $X$. Whenever $H$ is very ample, one has $g\left(-K_{X}\right)$ $\geq 3$ and $X$ is embedded as a threefold of degree $2 g-2$ in $\boldsymbol{P}^{g+1}$ by means of $|H|$. In particular, it follows that (3.5.3) gives an example of a pair 
$X, H$ where $H$ is not very ample. Note also that other examples where $H$ is not very ample arise from (3.2.3) when $d=1,2$ (see $[\mathrm{Mu}], 2.8$ ).

\title{
REFERENCES
}

[E] F. Enriques, Sui sistemi lineari di ipersuperficie algebriche ad intersezioni variabili iperellittiche, Math. Ann., 46 (1895), 179-199.

[I1] V. A. Iskovskih, Fano 3-folds I, Engl. trans. Math. U.S.S.R., Izv., 11 (1977).

[I2] - Fano 3-folds II, Engl. trans. Math. U.S.S.R., Izv., 12 (1978).

[K] S. Kleiman, Toward a numerical theory of ampleness, Ann. of Math., 84 (1966), 293-344.

[Ka] Y. Kawamata, Elementary contractions of algebraic 3-folds, Ann. of Math., 119 (1984), 95-110.

[M1] S. Mori, Threefolds whose canonical bundles are not numerically effective, Ann. of Math., 116 (1982), 133-176.

[M2] - Threefolds whose canonical bundles are not numerically effective, Algebraic Threefolds, Proceedings, Varenna 1981, Lecture Notes in Math., 947, SpringerVerlag (1982).

[M-M] S. Mori and S. Mukai, Classification of Fano 3-folds with $B_{2} \geq 2$, Manuscripta Math., 36 (1981), 147-162.

[Mu] J. P. Murre, Classification of Fano Threefolds according to Fano and Iskovskih, Algebraic Threefolds, Proceedings, Varenna 1981, Lecture Notes in Math., 947, Springer-Verlag (1982).

[R] M. Reid, Projective morphisms according to Kawamata, preprint, Univ. of Warwick, June 1983.

[Ro] L. Roth, Algebraic Threefolds, Springer-Verlag (1955).

[S1] A. J. Sommese, On the minimality of hyperplane sections of projective threefolds, J. reine angew. Math., 329 (1982), 16-41.

[S2] —- Ample divisors on 3-folds, Algebraic Threefolds, Proceedings, Varenna 1981, Lecture Notes in Math., 947, Springer-Verlag (1982).

\author{
Mauro Beltrametti \\ Ist. Matematico \\ Via L. B. Alberti, 4 \\ 16132 Genova \\ Italy \\ Marino Palleschi \\ Dip. di Matematica \\ Via C. Saldini, 50 \\ 20100 Milano \\ Italy
}

